\title{
Tables Showing the Monthly means of Meteorological Observations taken at Tiberias during 1907 and 1908
}

\section{Rasheed Nassar \& Elias Bisht}

To cite this article: Rasheed Nassar \& Elias Bisht (1910) Tables Showing the Monthly means of Meteorological Observations taken at Tiberias during 1907 and 1908, Palestine Exploration Quarterly, 42:1, 69-69, DOI: 10.1179/peq.1910.42.1.69

To link to this article: http://dx.doi.org/10.1179/peq.1910.42.1.69

曲 Published online: 20 Nov 2013.

Submit your article to this journal $\pi$

Џll Article views: 3

Q View related articles $\llbracket$ 
TABLES SHOWING THE MONTHLY MEANS OF METEOROLOGICAL OBSERVATIONS TAKEN AT TIBERIAS DURING 1907 AND 1908.

By Mr. Rasheed Nassar and Mr. Elias Bisht.

\begin{tabular}{|c|c|c|c|c|c|c|c|c|c|c|}
\hline \multirow{2}{*}{\multicolumn{3}{|c|}{$\begin{array}{c}\text { Monthly Means, } \\
1907 .\end{array}$}} & \multirow{2}{*}{ 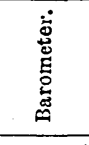 } & \multirow{2}{*}{$\begin{array}{l}\text { Att. } \\
\text { Ther. }\end{array}$} & \multicolumn{4}{|c|}{ Thermometers. } & \multicolumn{2}{|c|}{ Rain. } \\
\hline & & & & & Max. & Min. & $\begin{array}{c}\text { Dry } \\
\text { Bulb. }\end{array}$ & $\begin{array}{l}\text { Wet } \\
\text { Bulb. }\end{array}$ & Inches. & Days. \\
\hline January & $\ldots$ & $\cdots$ & $30 \cdot 927$ & $59 \cdot 5$ & $64 \cdot 3$ & $50 \cdot 5$ & $55 \cdot 8$ & $49 \cdot 5$ & $5 \cdot 04$ & 11 \\
\hline February & $\cdots$ & ... & $30 \cdot 731$ & 59 & $64 \cdot 8$ & $50 \cdot 5$ & $56 \cdot 4$ & $51 \cdot 4$ & $3 \cdot 21$ & 12 \\
\hline March & $\cdots$ & $\cdots$ & $30 \cdot 784$ & $55 \cdot 9$ & $65 \cdot 3$ & $54 \cdot 1$ & $57 \cdot 6$ & $51 \cdot 8$ & $2 \cdot 44$ & 9 \\
\hline April ... & $\cdots$ & $\ldots$ & $30 \cdot 728$ & $67 \cdot 5$ & $78 \cdot 4$ & 63 & $68 \cdot 5$ & 58 & $1 \cdot 10$ & 4 \\
\hline May ... & $\cdots$ & $\cdots$ & $30 \cdot 722$ & $76 \cdot 9$ & $91 \cdot 2$ & $68 \cdot 6$ & $76 \cdot 9$ & 65 & $\cdots$ & $\cdots$ \\
\hline June ... & $\cdots$ & ... & $30 \cdot 683$ & 82 & $95 \cdot 5$ & $69 \cdot 7$ & $81 \cdot 3$ & $69 \cdot 2$ & $\cdots$ & $\cdots$ \\
\hline July .. & $\ldots$ & ... & $30 \cdot 603$ & $87 \cdot 6$ & $98 \cdot 6$ & 72 & $83 \cdot 7$ & $74 \cdot 3$ & ... & $\cdots$ \\
\hline August & $\cdots$ & $\cdots$ & $30 \cdot 660$ & $82 \cdot 9$ & $98 \cdot 5$ & $71 \cdot 4$ & $84 \cdot 1$ & $74 \cdot 6$ & $\cdots$ & $\cdots$ \\
\hline September & $\cdots$ & ... & $30 \cdot 763$ & $82 \cdot 3$ & $90 \cdot 5$ & $67 \cdot 2$ & $79 \cdot 9$ & $66 \cdot 7$ & $\cdots$ & $\cdots$ \\
\hline October & $\cdots$ & ... & $30 \cdot 820$ & $77 \cdot 9$ & $80 \cdot 2$ & $66 \cdot 8$ & 76 & $64 \cdot 8$ & $1 \cdot 23$ & 2 \\
\hline November & $\cdots$ & $\cdots$ & $30 \cdot 900$ & 67 & $70 \cdot 5$ & $56 \cdot 3$ & $64 \cdot 5$ & $56 \cdot 4$ & $4 \cdot 62$ & 6 \\
\hline December & $\cdots$ & $\cdots$ & $30 \cdot 958$ & $62 \cdot 4$ & $67 \cdot 7$ & $51 \cdot 8$ & $59 \cdot 8$ & $53 \cdot 9$ & $3 \cdot 04$ & 6 \\
\hline Year & $\ldots$ & ... & $30 \cdot 772$ & $71 \cdot 7$ & 80.5 & $61 \cdot 8$ & $70 \cdot 4$ & $61 \cdot 3$ & $20 \cdot 68$ & 50 \\
\hline \multirow{2}{*}{\multicolumn{3}{|c|}{$\begin{array}{c}\text { Monthly Means, } \\
1908 .\end{array}$}} & \multirow{2}{*}{ 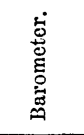 } & \multirow{2}{*}{$\begin{array}{l}\text { Att. } \\
\text { Ther. }\end{array}$} & \multicolumn{4}{|c|}{ Thermometers. } & \multicolumn{2}{|c|}{ Rain. } \\
\hline & & & & & $\operatorname{Max}$. & Min. & $\begin{array}{l}\text { Dry } \\
\text { Bulb. }\end{array}$ & $\begin{array}{l}\text { Wet } \\
\text { Bulb. }\end{array}$ & Inches. & Days. \\
\hline January & $\cdots$ & $\ldots$ & $30 \cdot 904$ & $58 \cdot 5$ & $62 \cdot 2$ & $49 \cdot 2$ & $55 \cdot 9$ & $50 \cdot 2$ & $4 \cdot 11$ & 12 \\
\hline February & $\cdots$ & $\cdots$ & $30 \cdot 917$ & $58 \cdot 3$ & $65 \cdot 6$ & $47 \cdot 3$ & $57 \cdot 3$ & $50 \cdot 8$ & $3 \cdot 74$ & 7 \\
\hline March & $\cdots$ & $\ldots$ & $30 \cdot 844$ & $63 \cdot 6$ & $73 \cdot 2$ & $51 \cdot 5$ & $62 \cdot 3$ & $54 \cdot 2$ & $1 \cdot 39$ & 3 \\
\hline April ... & $\cdots$ & $\cdots$ & $30 \cdot 403$ & 68 & $78 \cdot 9$ & $51 \cdot 6$ & $66 \cdot 9$ & $58 \cdot 4$ & 0.46 & 4 \\
\hline May ... & $\cdots$ & $\cdots$ & $30 \cdot 772$ & $76 \cdot 9$ & $92 \cdot 8$ & $63 \cdot 8$ & $76 \cdot 9$ & $65 \cdot 1$ & $\cdots$ & $\cdots$ \\
\hline June ... & $\cdots$ & $\cdots$ & $30 \cdot 696$ & $82 \cdot 1$ & $95 \cdot 2$ & $69 \cdot 5$ & $81 \cdot 5$ & $69 \cdot 8$ & $\cdots$ & $\cdots$ \\
\hline July $\ldots$ & $\cdots$ & $\cdots$ & $30 \cdot 608$ & $84 \cdot 8$ & $97 \cdot 5$ & 71 & $82 \cdot 9$ & $73 \cdot 2$ & $\ldots$ & $\cdots$ \\
\hline August & $\cdots$ & $\cdots$ & $30 \cdot 609$ & $86 \cdot 2$ & $98 \cdot 6$ & $72 \cdot 4$ & $84 \cdot 5$ & $74 \cdot 6$ & $\cdots$ & $\cdots$ \\
\hline Septe mber & $\cdots$ & $\cdots$ & $30 \cdot 738$ & $84 \cdot 3$ & $89 \cdot 4$ & $70 \cdot 5$ & $85 \cdot 2$ & $72 \cdot 5$ & $\ldots$ & $\cdots$ \\
\hline October & $\cdots$ & $\cdots$ & $30 \cdot 790$ & $79 \cdot 4$ & $88 \cdot 6$ & $64 \cdot 2$ & $76 \cdot 5$ & 69 & $\cdots$ & $\cdots$ \\
\hline November & $\cdots$ & $\cdots$ & $30 \cdot 860$ & $68 \cdot 8$ & $75 \cdot 7$ & $54 \cdot 9$ & $64 \cdot 8$ & 58 & $2: 35$ & 6 \\
\hline December & $\cdots$ & ... & $30 \cdot 903$ & 61 & $63 \cdot 6$ & $46 \cdot 2$ & $56 \cdot 9$ & $50 \cdot 8$ & $6 \cdot 30$ & 10 \\
\hline Year & $\cdots$ & $\cdots$ & $30 \cdot 753$ & $72 \cdot 6$ & $81 \cdot 7$ & $59 \cdot 3$ & $70 \cdot 9$ & $62 \cdot 2$ & $18 \cdot 35$ & 42 \\
\hline
\end{tabular}

